\title{
9 Conclusiones
}

En el presente estudio, hemos llevado a cabo una revisión pancrónica de un fenómeno de variación sintáctica que ha recorrido prácticamente toda la historia de la lengua española, y cuyo desenlace parece estar lejos de resolverse. Desde una perspectiva teórica y metodológica variacionista, para analizar la profundidad histórica del queísmo hemos comparado los patrones de variación en varios momentos de la historia. Para ello hemos contado con los materiales que proporcionan textos que, aun de naturaleza diversa, comparten una misma concepción oralizante de la lengua. Por un lado, un corpus histórico, que abarca un largo periodo de casi cinco siglos (comienzos del siglo XVI hasta la primera mitad del XX), y que se compone íntegramente de textos cercanos al polo de la inmediatez comunicativa, principalmente correspondencia privada. Por otro lado, tres corpus orales, representativos de otras tantas comunidades de habla peninsulares, con los que hemos evaluado el presente estado de lengua. En lo que sigue resumimos las principales implicaciones del estudio, así como las respuestas a las preguntas de investigación con las que iniciábamos estas páginas.

\subsection{La difusión del queísmo en la historia}

La primera conclusión que se deriva del trabajo es que las variantes queístas han sido la norma, antes que la excepción, a lo largo de la historia. La inserción de las preposiciones, que homologa algunas de estas construcciones con estructuras similares (tengo miedo de tu padre = tengo miedo de que vengas; trata de $t i=$ trata de que te vayas) tiene lugar a lo largo de un dilatado proceso diacrónico, no exento de prolongadas etapas de estabilización, y aun de rectificaciones y vueltas atrás. Durante buena parte del siglo XVI, el uso de las formas queístas fue prácticamente universal, si bien ya a partir del final de la centuria comienzan a detectarse algunos cambios, especialmente en el régimen de ciertas construcciones, que se intensificarán durante la centuria siguiente. Los siglos posteriores continúan esta misma senda, aunque, en general, de manera lenta y moderada, de tal forma que, tan solo en los albores del siglo XX, las inserciones preposicionales comienzan a aventajar globalmente a las elisiones. Sin embargo, este proceso parece estar experimentando una cierta reversión en las primeras décadas del presente milenio, al menos en determinados casos.

Ahora bien, esta síntesis ofrece una instantánea demasiado parcial, y en buena medida engañosa, de los hechos relacionados con esta variable lingüística. En primer lugar, porque la difusión de las soluciones queístas ha sido muy irregular en 
las diferentes estructuras sintácticas estudiadas. Así, de los tres casos de queísmo conjuntivo considerados, el que concierne a las completivas dependientes de sustantivos y adjetivos arroja históricamente un número mucho menor de variantes vernáculas que los otros dos, las completivas dependientes de verbos y las subordinadas introducidas por locuciones de diverso signo. Y no digamos respecto al queísmo pronominal en subordinadas de relativo, que, en términos generales, aparece sistemáticamente como el bloque sintáctico más proclive a las elisiones.

Por otro lado, la evolución del queísmo en cada una de estas construcciones ha experimentado también cambios que las singularizan. Así, las completivas dependientes de categorías nominales han ocupado siempre una posición inferior en la escala del queísmo, incluso en el siglo XVI, cuando las realizaciones vernáculas eran muy elevadas. Sin embargo, ya al final de esa centuria, y de manera más decidida a partir de las primeras décadas del siglo XVII, se inicia un pronunciado declive, y un claro movimiento favorable a las alternativas preposicionales. A partir de entonces, las frecuencias de uso se estabilizan durante un extenso periodo de tiempo, que abarca prácticamente los siglos XVIII y XIX. No obstante, el cambio favorable a las inserciones toma un nuevo impulso en la primera mitad del $\mathrm{XX}$, con cifras que se reproducen de nuevo en la etapa actual, cuando parece que asistimos a un nuevo periodo de estabilización del fenómeno. Sea como sea, tras cinco siglos de historia del español, las magnitudes cercanas al 80\% en el primer español clásico, descienden a una tercera parte (28\%) en el momento presente.

El comportamiento de las subordinadas dependientes de verbos, algunos de los cuales están íntimamente relacionados con elementos del grupo anterior (confiado, confianza, confiar; convencimiento, convencido, convencer, etc.) es, sin embargo, diferente. Por un lado, las proporciones de queísmo entre los verbos han estado siempre significativamente por encima de las de sustantivos y adjetivos. Por otro lado, su evolución diacrónica ha sido también distinta, y en ella destacan algunos vaivenes, acompañados de fases de estabilidad durante los periodos centrales, que probablemente den cuenta de mayores dosis de inseguridad en su construcción durante extensos periodos de la historia. Finalmente, y también a diferencia del bloque sintáctico anterior, en el momento actual continúa el ciclo desfavorable a la variante vernácula, excepción hecha de algunos usos idiosincrásicos con ciertos verbos (el caso de acordarse), cuyos patrones de variación-casi enteramente queístas-difieren extraordinariamente del resto.

El tercer y último tipo de queísmo conjuntivo analizado, el que revelan las oraciones introducidas por locuciones de diverso tipo (conjuntivas, preposicionales, adverbiales), presenta también perfiles distribucionales específicos. Por un lado, nos enfrentamos al queísmo que ha conocido una progresión lineal más clara, desde usos prácticamente categóricos en el primer español clásico hasta cifras que en cada periodo bajaban significativamente con respecto a las del ante- 
rior. De este modo, las fases de estabilización que hemos advertido en la historia de las otras construcciones no son visibles aquí. Aun así, los datos del momento actual apuntan hacia un moderado repunte de las soluciones queístas.

Finalmente, otra imagen singular ofrece el queísmo pronominal en las oraciones de relativo, al menos en las investigadas en el presente estudio: las subordinadas dependientes de sustantivos que encierran sentidos temporales y locativos. Además de tratarse del queísmo más persistente en la historia, su evolución muestra un patrón distribucional curvilíneo, con cifras muy elevadas en los Siglos de Oro, que descienden bruscamente en el siglo XVIII, para remontar de nuevo en las centurias siguientes, en un movimiento ascendente que continúa su curso en el momento actual.

\subsection{Diferencias en el condicionamiento interno de la variable}

Un estudio variacionista como el presente demuestra también que la singularidad exhibida por estos cuatro tipos sintácticos no se detiene en el plano distribucional, sino que alcanza también a su condicionamiento interno, esto es, al modo en que la selección de las variantes queístas se ha visto afectada en cada periodo por factores estructurales y extralingüísticos. Antes de valorar cómo se han comportado históricamente esos condicionantes en el fenómeno general del queísmo, conviene recordar cómo lo han hecho en cada una de las construcciones.

\subsubsection{Complementos de categorías nominales}

Si comenzamos este repaso por la incidencia de los factores lingüísticos entre los complementos de categorías nominales, cabe destacar en primer término que la variación se ha mantenido sistemáticamente al margen de algunos condicionantes como la polaridad (afirmativa/negativa), el priming discursivo (preposición, que) o el tipo de preposición implicada (de/otras). En efecto, contrariamente a las hipótesis de partida (§3.2), ninguno de ellos ha sido seleccionado por el estadístico de regresión en periodo alguno, ni en el corpus histórico, ni tampoco en el corpus oral contemporáneo. Tampoco lo ha sido la persona del verbo en aquellos sustantivos y adjetivos que aparecen habitualmente en determinadas estructuras colocativas (caber duda) o atributivas (estar seguro), un resultado muy diferente al que se obtiene entre los complementos verbales, y al que nos referiremos más adelante. De este modo, las ideas formuladas por varios autores acerca del efecto distanciador de las inserciones preposicionales (ver supra §2.2.1 y 3.2.7) no se han visto confirmadas en la historia del español, al menos en esta categoría sintáctica. 
Otros factores han demostrado cierta incidencia, aunque tímida, y limitada a algunos momentos de la historia. Por ejemplo, la frecuencia de las unidades en el discurso ha sido avalada en el periodo moderno (1700-1835) en el sentido previsto (ver supra §3.2.4): el queísmo fue entonces más intenso entre las estructuras más recurrentes que entre las más esporádicas. Por su parte, el grado de adyacencia muestra su poder explicativo tan solo en el corpus oral, con un número significativamente mayor de elisiones cuando el núcleo de la construcción y el nexo que aparecen separados (no cabe duda, queridos amigos, que...), un resultado que podría justificarse por las mayores dificultades para construir la rección preposicional cuando estos elementos se hallan a distancia. Ahora bien, ni la frecuencia ni el grado de adyacencia son seleccionados en otras etapas de la historia, aunque en el caso de este último a ello han podido contribuir algunas insuficientes muestrales en los entornos no adyacentes, francamente minoritarios en el corpus. De hecho, en todos los periodos, la dirección del efecto ha sido siempre la misma en estas construcciones: los contextos no adyacentes superan en usos queístas a los adyacentes.

Más relevancia han demostrado otros predictores cuyo condicionamiento sobre la variación ha actuado con nitidez durante periodos más extensos de la historia. Entre estos destaca el modo verbal de la subordinada. Ya desde el periodo clásico, y en un proceso que no se detendrá hasta nuestros días, las soluciones queístas se han visto favorecidas sistemáticamente en las combinaciones con el indicativo, mientras que la conexión con el subjuntivo ha sido invariablemente un entorno menos propicio. El hecho de que en dos etapas intermedias (siglo XVII y periodo moderno) el estadístico de regresión no haya seleccionado el predictor-frente a lo que sí ocurre en los extremos-no oculta que, también en ellos, las diferencias frecuenciales apuntan en la misma dirección. Como vimos en su momento (ver supra §3.2.2), esta relación se ha vinculado con un cierto valor icónico de las preposiciones, que aparecerían con más frecuencia en aquellos contextos en los que el compromiso del hablante con la veracidad de sus enunciados es menos sólido, como sucede a menudo con los valores asociados al subjuntivo. Justo lo contrario de lo que ocurre con el indicativo, lo que podría justificar la menor necesidad de las preposiciones en las combinaciones con este modo.

Otro factor que opera prácticamente a lo largo de toda la serie histórica es el grado de determinación. Limitado esta vez al estudio de los sustantivos, la distinción entre aquellos nombres que van precedidos por el artículo determinado y los que no muestra un condicionamiento sostenido en el tiempo. Sin embargo, contrariamente esta vez a nuestras expectativas iniciales (ver supra §3.2.12), los núcleos de la construcción precedidos por el artículo han figurado siempre como un entorno que desalienta las soluciones queístas. 
Con todo, el predictor más robusto y estable en el tiempo es el que parte del grado de fusión con un verbo. A lo largo de cinco siglos, la participación de estas unidades en estructuras de carácter colocativo o semilocucional, bien con un verbo soporte (el caso de los sustantivos), bien en construcciones atributivas (adjetivos), ha favorecido invariablemente las soluciones queístas, frente a aquellos contextos en los que estas categorías nominales se ven libres de tales ataduras sintáctico-semánticas. Y aunque el momento actual muestra un ligero estrechamiento de los pesos explicativos entre ambos contextos, lo cierto es que continúa siendo un robusto predictor del queísmo en el presente estado de lengua. Como en algunos estudios llevados a cabo en comunidades de habla contemporáneas (ver supra §3.2.10), las hipótesis de partida se cumplen esta vez, y justifican que la especial conexión entre sustantivos, adjetivos y verbos ha podido conducir históricamente a la interpretación de tales estructuras sintácticas más como verbales-y, por tanto, más favorables históricamente al queísmo-que como auténticamente nominales.

\subsubsection{Complementos verbales}

Al igual que en el grupo anterior, el queísmo en las completivas dependientes de verbos ha mostrado un sistemático rechazo a la influencia de ciertos predictores. Así, la distinción entre verbos pronominales y no pronominales, que en ocasiones se ha propuesto como determinante-con mayores índices de queísmo entre los primeros que entre los segundos (ver §3.2.1) - , no ha revelado apenas diferencias, y mucho menos significativas, en periodo alguno de la historia. Tampoco lo hacen factores sintácticos como el grado de adyacencia o la distancia entre el núcleo de la construcción y el nexo que. El hecho de que estos aparezcan juntos (trato (de) que viniera) o a distancia (trató con toda su buena voluntad (de) que) no demuestra en esta ocasión ninguna relevancia, contrariamente a nuestros planteamientos iniciales, y a lo que hemos advertido entre los complementos nominales en el apartado anterior. Del mismo modo, las diferencias entre el presente de indicativo, sobre cuyos eventos el hablante puede tener un mayor control (lo que debería favorecer las elisiones según las tesis funcionalistas), y las formas del pasado o el futuro, en las que ese control se supone menor (lo que alentaría los usos preposicionales), han sido sistemáticamente descartadas en el eje diacrónico, contrariamente también a las hipótesis de partida (ver supra §3.2.8).

Más interés han demostrado, sin embargo, otros predictores, cuyo poder explicativo se ha dejado sentir en diversos momentos de la historia. En el caso del modo verbal de la subordinada, la dirección del efecto coincide, además, con la observada entre los complementos nominales. También ahora el queísmo se 
ve favorecido en la conexión con el indicativo y desfavorecido en las combinaciones con el subjuntivo. Sin embargo, a diferencia del bloque anterior, el factor va perdiendo robustez con el paso del tiempo: es seleccionado en el español clásico y más débilmente en el periodo moderno, pero pierde el aval estadístico en la primera mitad del siglo XX, confirmándose este mismo resultado en el presente estado de lengua. Aun así, de su poder en el pasado quedan todavía algunos vestigios, bajo la forma de diferencias frecuenciales que, aun siendo ya de menor entidad, se siguen moviendo en la misma dirección.

Un segundo predictor que ha ido perdiendo solidez en la historia de la lengua es la frecuencia de las construcciones en el discurso. Conforme a lo previsto inicialmente, hemos advertido periodos (clásico y moderno) en los que el nivel de queísmo era directamente proporcional a la recurrencia de las construcciones. En estas etapas, las soluciones vernáculas eran mayores en las construcciones verbales más frecuentes que en aquellas que aparecen de forma más ocasional en el discurso, lo que convertía a estas últimas en un vector favorable para las inserciones preposicionales. Ahora bien, tanto en el periodo contemporáneo como en el corpus oral presente tales restricciones dejan de funcionar.

El condicionamiento ejercido por la persona del verbo muestra un signo muy diferente en este grupo de construcciones y en el anterior. Si los patrones de variación en las completivas dependientes de sustantivos y adjetivos que participan en colocaciones verbales eran completamente ajenos a la incidencia de este predictor (ver supra §9.2.1), la persona exhibe ahora un papel mucho más relevante, sobre todo a partir del español moderno. Con todo, su comportamiento a lo largo de la historia permite vislumbrar algunos cambios significativos, tanto en la dirección del efecto, como en el papel que cada persona ejerce en la variación. De hecho, el único resultado común a toda la serie histórica reside en la sistemática mayor aversión por las soluciones queístas en las combinaciones con la 3. a persona, que, como contrapartida, se convierte históricamente en la principal aliada de las variantes preposiciones. Este resultado se ajusta bien a las hipótesis funcionales que han vinculado fenómenos como el queísmo y el dequeísmo con el valor icónico de estas partículas (ver §2.2.1 y §3.2.7). Así, tanto en uno como en otro fenómeno, se ha propuesto que las inserciones preposicionales tienen un mayor peso en aquellos contextos sobre los que el hablante posee un menor control, ya sea en términos de evidencialidad, ya en otros sentidos más subjetivos y no siempre fáciles de categorizar. Sea como sea, no cabe duda de que la 3. a persona (la no persona del acto comunicativo) ocupa el extremo más débil en esa escala de control.

Ahora bien, tanto la relevancia del factor, como, sobre todo, la dirección explicativa en su interior muestran algunas particularidades dignas de mención. En primer lugar, hay que resaltar el hecho de que, en el corpus histórico, el predictor no ha sido seleccionado en su estructura tripartita en ningún momento. 
Tan solo en el corpus oral contemporáneo los resultados se ajustan a lo previsto inicialmente: en cabeza de las soluciones queístas aparece la 1. ${ }^{a}$ persona, que identifica al hablante, fuente principal de información objetiva, seguida por la 2. ${ }^{a}$ persona, la del interlocutor, y con la ya mencionada 3. ${ }^{a}$ persona como el contexto menos favorable a las elisiones. Sin embargo, este esquema no se observa en ningún otro momento de la historia.

De hecho, el factor solo resulta significativo en los periodos moderno y contemporáneo, y tan solo a partir de una reestructuración binaria, que, además, es diferente en cada fase. Así, en el español moderno, la posición privilegiada para las soluciones queístas es ocupada por la persona del hablante, pero la incidencia negativa es compartida tanto por la $2 .^{\text {a }}$ como por la $3 .^{a}$ persona. Sin embargo, en el periodo contemporáneo, además de debilitarse-tan solo es seleccionado en uno de los análisis de regresión, no en el que toma en conjunto los dos predictores aleatorios-, la distribución de estos valores cambia: el contexto más favorable para las elisiones es compartido por la $1 .^{\mathrm{a}} \mathrm{y}$ la $2 .^{\mathrm{a}}$ persona, al tiempo que la 3. ${ }^{\mathrm{a}}$ queda como único contexto desfavorable.

En definitiva, los resultados parecen apuntar a una reorganización progresiva de este condicionante en la historia, que tan solo en tiempos recientes muestra una dirección del efecto enteramente ajustada a las hipótesis mencionadas. Aun así, quedaría todavía por explicar por qué ninguno de esos efectos se advierte en las estructuras colocativas integradas por verbo + sustantivo o adjetivo, cuyas restricciones morfosintácticas son similares a las verbales.

Algo similar ocurre con la preposición implicada en la variación. A diferencia también de las completivas dependientes de sustantivos y adjetivos, con los que algunos de estos verbos mantienen estrechas relaciones formales y semánticas, los regímenes preposicionales que seleccionan de sistemáticamente han favorecido durante largos periodos de la historia el queísmo, mientras que aquellos en los que se ven concernidas otras preposiciones (en, con, $a$ ) lo han hecho en una proporción significativamente menor. Cabe especular con la posibilidad de que la persistencia cognitiva que aportan los contextos más frecuentes (como lo son, de hecho, las combinaciones con de) o la mayor desemantización de esta partícula estén detrás de estos resultados, pero de nuevo no explica por qué no tiene el mismo efecto en otras categorías relacionadas.

Finalmente, el corpus oral nos ha permitido descubrir también la incidencia leve de un predictor discursivo desconocido hasta el presente estado de lengua. En efecto, frente a su irrelevancia en las centurias anteriores, en los albores del siglo XXI se advierte el poder disimilatorio de la presencia en el contexto inmediatamente anterior de la misma preposición implicada en el régimen verbal. De esta manera, y por razones que se antojan eufónicas, en tales entornos los niveles de queísmo son significativamente mayores. 


\subsubsection{Locuciones}

Las diferencias estructurales entre estas construcciones y las reseñadas hasta el momento justifican, probablemente, que el condicionamiento del queísmo entre las locuciones exhiba también algunas singularidades. Por un lado, nos encontramos ante el bloque sintáctico cuyas restricciones internas son más limitadas, lo cual no impide que algunas muestren una notable fortaleza a lo largo de la historia. Así ocurre con el factor tipológico, que distingue entre las locuciones que se originan a partir de un núcleo nominal (a pesar (de) que, a (fin) de $q u e . .$.$) y las que se construyen mediante el auxilio de otras categorías, como el$ adverbio (después (de) que, antes (de) que...). En todos los periodos examinados, incluido el actual, las soluciones queístas han sido siempre más numerosas en estas últimas que en las primeras. Por otro lado, las contribuciones de ambos contextos en cada periodo son parecidas, lo que avala su consistencia histórica, y hace prever el mantenimiento de su poder explicativo a medio y largo plazo. Estos resultados pueden guardar relación con diferencias en el grado de gramaticalización de estas construcciones. De ser ello así, este sería menor en las locuciones derivadas de sustantivos, lo que justificaría un menor grado de fusión con el nexo que y, por tanto, la necesidad de contar con un enlace suplementario, como la preposición. Por el contrario, esa gramaticalización estaría más avanzada en los demás casos, lo que podría explicar la menor necesidad de otros enlaces al margen de la conjunción.

El queísmo conjuntivo en las locuciones muestra algunos condicionamientos ya advertidos en otras categorías sintácticas, si bien ahora la dirección del efecto resulta más errática. Así ocurre, por ejemplo, con el modo verbal de la subordinada, un factor que vuelve a dejar su huella en el fenómeno que nos ocupa. Sin embargo, a lo largo de la historia, la dirección explicativa que muestran sus datos es, en buena medida, contraria a la que habíamos visto hasta el momento. Aunque el predictor tan solo es seleccionado por la regresión en el periodo contemporáneo, las diferencias frecuenciales en el español clásico y en el moderno apuntan en todos los casos a una prevalencia de las variantes queístas en combinación con el subjuntivo, justo al revés de lo que habíamos visto en las completivas dependientes tanto de categorías nominales como verbales. Por el contrario, el corpus oral representativo de la presente centuria arroja resultados que se aproximan más a los hallados en estas últimas. En definitiva, el poder explicativo del factor parece haber cambiado de rumbo en tiempos recientes, cuando su perfil distribucional se ha asimilado al de otras estructuras del queísmo conjuntivo.

Finalmente, también en el momento actual actúa por primera vez un factor posicional cuya incidencia se había demostrado irrelevante en el pasado. Y así, los resultados del corpus oral descubren que la posposición de las subordinadas 
introducidas por la locución con respecto a la oración con la que están relacionadas favorece las expresiones queístas, frente a mayores reticencias de la variante elidida en la posición antepuesta.

\subsubsection{Queísmo pronominal}

Durante más de 500 años, la variación en las oraciones de relativo dependientes de sustantivos que encierran sentidos temporales y locativos se ha visto condicionada sistemáticamente por la función sintáctica del antecedente. En particular, el papel de complemento circunstancial de este ha representado siempre uno de los contextos más favorables para las variantes queístas. No obstante, aunque esta dirección del efecto se mantiene inalterable durante toda la serie histórica, en los primeros periodos (clásico y moderno) el predictor interactúa con otro factor sintáctico, la distinción entre oraciones especificativas y explicativas, un cruce cuyo sentido cambia, además, de una época a otra. Así, aunque la combinación entre la función circunstancial y la oración especificativa aparece en ambas etapas como el entorno más favorable a las elisiones, el periodo moderno pone ya el foco sobre el papel primario de la función sintáctica, dejando en un segundo plano el tipo de oración. De hecho, este movimiento continuará en el futuro, de tal manera que, ya en la primera mitad del siglo XX, el factor tipológico pierde toda relevancia, un hecho que se confirma igualmente en los resultados del corpus oral contemporáneo.

Aunque lejos de la importancia que muestra la función sintáctica del antecedente, el queísmo pronominal se ha revelado también sensible en varios momentos de la historia a la incidencia de otros factores lingüísticos. El grado de adyacencia es uno de ellos, como demuestran los datos de las etapas clásica y moderna. En ambos periodos, la posición adjunta del antecedente y el pronombre de relativo ha favorecido las elisiones, al contrario que la presencia de otras palabras entre ambas unidades. Sin embargo, el factor pierde fuerza en el periodo contemporáneo y, aunque las diferencias frecuenciales siguen apuntando en la misma dirección, estas son ya de menor entidad y pierden su aval estadístico. Y este debilitamiento se extrema en la actualidad, cuando la diferencia entre ambos contextos sintagmáticos queda ya completamente neutralizada.

Del mismo modo cabe hablar del paralelismo estructural (priming) ejercido por la presencia de la preposición (mayoritariamente en, esta vez) en el contexto previo. Así, en el periodo contemporáneo, se advierte la influencia positiva de esta presencia en el uso posterior de esa misma preposición. Por el contrario, en el momento actual el factor se debilita y deja de ser seleccionado por el estadístico de regresión. 
La frecuencia de las construcciones en el discurso ha dejado también su huella en el queísmo pronominal. Durante buena parte de la historia se detecta una influencia positiva de este predictor en las elecciones queístas, y, además, en el sentido previsto: cuanto más recurrentes son esas estructuras en los textos, más abundan las variantes cero, y viceversa. Esta capacidad explicativa se refuerza incluso en el español actual, frente a una incidencia más tímida en el pasado.

Finalmente, en el presente estado de lengua actúa también por primera vez un factor estructural que no había dejado huella en las fases anteriores. Frente a la relevancia histórica que el modo de la subordinada ha mostrado en otros casos de queísmo, su influencia en las oraciones de relativo es mucho más restringida, y se limita ahora al corpus oral contemporáneo. Y aunque en este periodo el rango del predictor no es muy elevado, muestra una dirección del efecto que hemos visto ya de manera recurrente: las combinaciones con el indicativo favorecen moderadamente la variante cero, al tiempo que el subjuntivo la desfavorece en similar medida.

\subsection{Variaciones históricas en el efecto de los condicionantes estructurales}

De lo visto hasta aquí se desprende que, con pocas excepciones, son más las fuerzas estructurales que separan a los diferentes tipos de queísmo analizados en estas páginas que aquellas que las unen.

Tres de las cuatro construcciones estudiadas muestran una conexión indeleble con un predictor privativo, cuya fuerza explicativa se confirma en todos los periodos estudiados y, además, con la misma dirección del efecto en su interior. En el caso de las completivas dependientes de sustantivos y adjetivos ese factor atiende al grado de fusión de estas unidades con un verbo. A lo largo de más de cinco siglos, desde el primer español clásico hasta la actualidad, tanto los sustantivos que se combinan con un verbo soporte como los adjetivos que se construyen con verbos atributivos han visto un plus de variantes queístas. Por el contrario, las unidades nominales que aparecen en contextos sintáctico-semánticos menos trabados han representado siempre un importante acicate para la difusión de las inserciones preposicionales. Asimismo, hemos visto cómo los pesos explicativos de ambos contextos son muy similares en todas las fases históricas, lo que augura que el predictor seguirá condicionando fuertemente estas construcciones en el futuro.

Por lo que al grupo de las locuciones se refiere, este papel protagonista corresponde a un factor completamente distinto: el origen categorial de la unidad que actúa como base de la locución. A este respecto, hemos comprobado cómo, 
en toda la serie histórica, las locuciones más proclives a las soluciones queístas son aquellas que se forman a partir de un elemento no sustantivo. Por el contrario, las inserciones preposicionales se han difundido prioritariamente en las construcciones cuyo núcleo original es un nombre. Este resultado, que, como hemos visto, refleja probablemente el menor grado de gramaticalización de estas últimas locuciones frente a las anteriores, se deja sentir igualmente en todo el eje temporal, y además con valores muy extremos en todos los periodos. Todo ello confirma la extraordinaria profundidad histórica del factor, y lleva a presagiar que la variación en el seno de las locuciones continuará viéndose condicionada por él durante mucho tiempo.

Por su parte, el queísmo pronominal en las oraciones de relativo exhibe también una conexión persistente con otro factor sintáctico: la función del antecedente. Como hemos tenido ocasión de comprobar, en todas y cada una de las etapas analizadas, las variantes queístas han sido especialmente frecuentes en aquellos casos en los que la función del antecedente en la oración matriz es la de complemento circunstancial, mientras que las elisiones han disminuido siempre de manera significativa en el resto de funciones sintácticas. Y, aunque en los periodos clásico y moderno el factor opera en combinación con el tipo de relativa (especificativa/explicativa), a partir de 1700 su papel será cada vez más relevante (orillando al anterior), como demuestra su selección en solitario en las etapas posteriores, incluido el presente estado de lengua.

Por el contrario, el grupo de los complementos verbales se mantiene al margen de cualquier alianza con un predictor sistemático en el transcurso de la historia. El que más se le aproxima es la persona del verbo, aunque, a diferencia de los reseñados hasta ahora, este muestra un comportamiento más variable. Además de no ser seleccionado en el periodo clásico, y hacerlo de una manera débil en el contemporáneo, el factor exhibe algunos cambios en su dirección explicativa con el paso tiempo. De este modo, hemos comprobado cómo el papel protagonista en las elisiones no ha estado siempre ocupado por la $1 .^{\mathrm{a}}$ persona, cuyo liderazgo en varios momentos de la historia ha compartido con la 2. ${ }^{a}$ persona. Sea como sea, en lo que sí coincide toda la serie histórica es en la mayor aversión hacia las soluciones queístas entre las formas de la $3 .^{a}$ persona, y, por tanto, el mayor aliento de estas a las inserciones preposicionales. Al final, tan solo en el corpus actual se confirma la escala funcional que en las últimas décadas ha vinculado este factor con principios como la eventualidad, y que establece una clara jerarquía entre las tres personas del acto comunicativo en la selección de las formas queístas (ver supra §3.2.7).

Junto a esta particular conexión de cada tipo de queísmo con un factor estructural privilegiado, los resultados del estudio revelan también algunas irregularidades que confirman la particular idiosincrasia del fenómeno en cada bloque 
sintáctico. Así, hemos advertido, por ejemplo, cómo ciertos factores operaban significativamente en determinadas construcciones, pero no lo hacían en otras.

La persona es, de nuevo, uno de ellos. Determinante, como hemos visto, entre las completivas dependientes de verbos, su papel se diluye completamente en las construcciones nominales que funcionan con un verbo soporte o en construcciones atributivas. Paradójicamente, pues, el factor se presenta decisivo para explicar la variación en verbos como avisar o asegurarse, pero no así en construcciones verbales relacionadas, como dar aviso o estar seguro.

Un desenlace inverso ocurre con la preposición implicada en la construcción: los verbos que rigen sistemáticamente la partícula de han favorecido el queísmo en mayor medida que aquellos que subcategorizan otra preposición. Sin embargo, este hecho sintáctico es ahora completamente irrelevante entre las completivas dependientes de sustantivos y adjetivos. Como contrapartida, el tipo de determinación surge como uno de los factores más consistentes en la explicación del queísmo en estas construcciones. Las variantes queístas aparecen sistemáticamente más entre los sustantivos precedidos por el artículo determinado, un condicionamiento que ha mostrado una notable fortaleza en todos los periodos de la historia estudiados, en los que ha sido invariablemente seleccionado por el estadístico de regresión. Sin embargo, este mismo factor no revela incidencia alguna en el queísmo pronominal, de tal manera que la presencia o no del artículo determinado con el antecedente de la oración de relativa no nos dice nada en esta ocasión.

Finalmente, y aunque esta vez la relevancia del factor haya sido históricamente menor, también cabe incluir en esta nómina a uno de los tipos de priming estudiados, el que atiende a la presencia en el cotexto previo del nexo que. Aunque solo haya sido seleccionado por la regresión en el periodo actual, las diferencias frecuenciales han mostrado siempre un particular efecto disimilatorio, según el cual esa presencia desalienta el queísmo y favorece, por el contrario, la variante preposicional. Sin embargo, esa restricción tan solo se manifiesta entre los complementos verbales, sin que se aprecie nada similar en las otras tres categorías estudiadas.

Ciertamente, hemos descubierto también algunos predictores cuya influencia se advierte en más de una categoría sintáctica, pero incluso en estos casos la dirección del efecto no siempre ha sido coincidente. Así ocurre, por ejemplo, con el modo verbal de la subordinada, uno de los condicionantes más consistentes y sistemáticos en la explicación del queísmo a lo largo de la historia. Tanto en las completivas dependientes de categorías nominales como en las subordinadas subcategorizadas por verbos, las variantes elididas se han visto favorecidas durante largos periodos en las combinaciones con el indicativo, frente a la mayor resistencia del subjuntivo. Con todo, la fortaleza de este factor se ha revelado 
mayor entre las primeras, ya que su poder predictivo llega hasta el momento presente. Por el contrario, entre los complementos verbales se observa un progresivo debilitamiento del factor con el paso del tiempo, que queda ya completamente neutralizado a comienzos del siglo XXI en el corpus oral.

Lo contrario ocurre, sin embargo, en el queísmo pronominal, que se ha mantenido al margen de estas presiones estructurales durante la mayor parte de la historia, lo que no ha impedido que el factor sea seleccionado en el presente estado de lengua, y con la misma dirección del efecto reseñada. Con todo, más singular es aún la incidencia de este factor entre las locuciones. Aunque el momento presente se suma a esta tendencia pancrónica al favorecimiento del queísmo en las subordinadas en indicativo, la dirección del efecto en el resto de la serie histórica ha apuntado justamente en la dirección opuesta. De este modo, las variantes elididas han sido casi siempre más numerosas en combinación con el subjuntivo que con el indicativo.

La misma divergencia se aprecia en relación con otro factor cuya relevancia se ha comprobado en varias categorías y momentos de la historia, el grado de adyacencia entre el núcleo de la construcción y el nexo que. Descartado, como se recordará, en el análisis de las locuciones (dada la ausencia de contextos no adyacentes), y sin relevancia alguna entre los complementos verbales, las dos construcciones afectadas por el predictor han sido exclusivamente las completivas dependientes de categorías nominales y las oraciones de relativo. Sin embargo, su influencia en ambas ha sido muy diferente. Así, en las oraciones relativas, las variantes queístas han predominado históricamente en los contextos de adyacencia, mayoritarios en el discurso, mientras que las inserciones preposicionales se han ido haciendo un hueco en aquellos casos en los que se insertan otras palabras entre ambas unidades. Aun así, la mayor robustez del predictor se ha dejado sentir sobre todo en las etapas clásica y moderna, mientras que se ha mostrado ya bastante más debilitado en el periodo contemporáneo, al tiempo que se neutraliza por completo en el momento actual. Por el contrario, la dirección explicativa del factor en el queísmo conjuntivo asociado a los complementos nominales ha sido justamente la inversa, de tal manera que esta vez los entornos adyacentes han mostrado casi siempre menores dosis de queísmo que los no adyacentes.

Al final, tan solo la frecuencia de las estructuras en el discurso muestra una incidencia más uniforme. Según esta, y aunque con diferente intensidad y poder explicativo en distintos momentos de la historia, las variantes queístas se han visto reforzadas en las estructuras más recurrentes.

En suma, los resultados del estudio demuestran que tanto la extensión como el condicionamiento interno del queísmo han diferido considerablemente en cada una de las categorías sintácticas investigadas. Veamos ahora qué conclusiones se derivan de los factores extralingüísticos examinados en el estudio. 


\subsection{La incidencia de los factores extralingüísticos}

Frente a lo que acabamos de ver, los datos extralingüísticos muestran una considerable mayor uniformidad, como demuestra la sistemática selección (y no selección) de los mismos factores en prácticamente todas las construcciones analizadas.

Si comenzamos este repaso por aquellos que se han revelado más importantes, debemos hacerlo por el que ha demostrado una influencia más decisiva, el estatus social. Concebido a partir de una división tripartita de la sociedad en todos los periodos, los resultados del estudio demuestran que todas las construcciones estudiadas se han visto condicionadas por este factor en algún momento de la historia e, invariablemente, con la misma dirección del efecto: las variantes queístas han sido lideradas por los representantes de las clases bajas, y desfavorecidas por los miembros de las élites sociales y culturales, al tiempo que los estratos medios quedaban casi siempre en una posición intermedia.

Aun así, la relevancia del factor no ha sido idéntica en todas las construcciones, ni en todas las etapas investigadas. La mayor fortaleza del predictor se ha demostrado en el queísmo pronominal, donde ha sido seleccionado por la regresión desde el español clásico hasta el momento actual. La misma dirección explicativa se comprueba entre los complementos de categorías nominales, si bien, en esta ocasión, el factor es todavía descartado en el corpus del siglo XVI, cuando las variantes queístas representaban la norma en todo el espectro social. Sin embargo, ya a partir del XVII, las tendencias apuntadas se consolidan en una distribución sociolectal que permanecerá hasta nuestros días. Este perfil es menos robusto entre los complementos verbales, en los que el predictor tan solo es seleccionado en los dos periodos más recientes, si bien ya en el español clásico y en el periodo moderno las diferencias frecuenciales se movían en la misma dirección. Aun así, son las locuciones el grupo sintáctico en el que menos incidencia tiene el factor, con largos periodos de la historia ajenos a este perfil sociolectal, al que tan solo se incorpora en las etapas más recientes.

Las claras diferencias diastráticas observadas no se extienden, sin embargo, al sexo de los hablantes, pese a la relevancia demostrada por este factor en tantos fenómenos de variación y cambio lingüístico. Ahora bien, las conclusiones en torno a este factor se ven lastradas en el estudio por las extraordinarias diferencias en la representación de hombres y mujeres en el corpus histórico (no así en el corpus oral contemporáneo), especialmente acusadas en algunas fases históricas, como sucede en la muestra del español moderno. Con estas limitaciones metodologógicas, la regresión tan solo arroja como significativas las diferencias entre hombres y mujeres en las construcciones nominales del siglo XVII, un momento clave, como hemos comprobado, en la difusión de las variantes preposiciona- 
les dentro de esta categoría. En esta centuria, vemos a las mujeres como claras impulsoras de las formas novedosas, en línea con lo observado repetidamente en la sociolingüística en los procesos de cambio hacia nuevas normas de prestigio. Frente a ellas, los hombres se muestran más fieles a las variantes vernáculas. En todos los demás casos, sin embargo, las diferencias entre unas y otras son descartadas por la regresión. A ello ha podido contribuir, como hemos indicado, los mencionados desequilibrios muestrales, de ahí que quepa plantearse qué hubiera podido ocurrir de contar con muestras más equilibradas. Esta pregunta es, sin duda, legítima, aunque no puede esconder el hecho de que el factor ha sido también descartado en el corpus oral, en el que la representación es esta vez mucho más equitativa, y en el que las diferencias entre hombres y mujeres en las frecuencias del queísmo son mínimas, y en ningún caso significativas en las cuatro construcciones analizadas. En definitiva, la cuestión que cabe plantearse es si estos resultados, que revelan una notable uniformidad generolectal en la distribución del queísmo en el español peninsular contemporáneo, son trasladables a otros periodos de la historia.

$\mathrm{Al}$ igual que el estatus social, la variación analizada en estas páginas se ha visto fuertemente condicionada por el tenor comunicativo. Limitado esta vez al corpus histórico, este factor estilístico se ha configurado mediante el cruce entre dos parámetros: la temática de los textos y el tipo de relación entre los participantes en los ejes del poder y la solidaridad. Los resultados del estudio muestran una mayor incidencia del queísmo en los textos más espontáneos y de concepción más oralizante, característicos, sobre todo, de las cartas privadas entre miembros de la unidad familiar a los que unen estrechos vínculos afectivos. En el extremo opuesto se sitúan, también de manera invariable, los textos más alejados de ese polo de la inmediatez comunicativa, como la correspondencia de contenido menos personal entre individuos alejados en los mencionados ejes de la solidaridad y el poder.

Como en el caso del estatus, también ahora el tipo de queísmo que se ha revelado más sensible a este factor estilístico ha sido el pronominal, al haber sido seleccionado por la regresión en dos de las etapas consideradas (español moderno y contemporáneo), si bien ya en el español clásico se aprecian algunas diferencias de menor entidad en este mismo sentido. Menos contundentes son los resultados entre los complementos de categorías nominales, donde la jerarquía estilística es solo seleccionada en el español contemporáneo, si bien, ya desde el siglo XVII, se advierte una tendencia a la distinción entre los contextos más cercanos-y proclives al queísmo-y los demás puntos del eje estilístico. Y lo mismo cabe decir de los complementos verbales, entre los que la jerarquía reseñada es avalada de nuevo tan solo por el estadístico de regresión en el español contemporáneo, aunque ya desde el español clásico se asiste a una especial inclinación por la variante vernácula en los entornos más espontáneos. 
En el plano dialectal, sin embargo, las conclusiones son mucho más conservadoras, pero no por ello menos interesantes, ya que los datos disponibles reflejan una notable uniformidad a lo largo de la historia. En este sentido, ni la oposición entre variedades norteñas y centro-meridionales, por un lado, o entre occidentales y orientales, por otro, han ofrecido datos reveladores. Aunque las lagunas muestrales existentes en ambos casos nos han impedido incluir ambas clasificaciones en el análisis de regresión junto con el resto de predictores, las diferencias frecuenciales halladas han sido, por lo general, escasas y, lo más importante, no han demostrado ningún patrón distribucional uniforme en ninguna de las construcciones analizadas. Por otro lado, estos resultados se ven confirmados en el corpus actual, donde el predictor sí es incluido en la regresión, pero cuya influencia-limitada a la distinción entre variedades occidentales y orientales-se ha visto completamente descartada. De paso, ello nos ha permitido poner en duda la influencia del contacto de lenguas con el catalán como un elemento determinante en la distribución del queísmo, contrariamente a lo que se ha propuesto en alguna ocasión (ver supra §3.3.5).

Poco revelador se ha demostrado, asimismo, el contexto migratorio, un factor que en el corpus histórico nos ha llevado a distinguir entre los textos escritos por españoles con destino en América, en condiciones normalmente caracterizadas por un mayor contacto interdialetal y favorables, por tanto, a procesos de simplificación o koineización, y textos escritos desde España, en entornos sociales generalmente más convencionales. El predictor ha sido rechazado sistemáticamente, lo que no oculta que, en la selección de las variantes queístas, el contexto americano haya ido generalmente por delante, al menos en tres de las cuatro construcciones estudiadas (una excepción son las locuciones). Aun así, estas diferencias han sido generalmente de escasa entidad, de ahí que, con la muestra disponible, no podamos aventurar conclusiones más arriesgadas. Por otro lado, el hecho de que tan solo en la etapa contemporánea estas diferencias adquieran una entidad algo mayor podría sugerir la progresiva asimilación de los emigrantes a unas variedades americanas en las que la dialectología contemporánea ha visto una mayor inclinación por el queísmo que en las peninsulares (ver supra §2.4). Ello sería un argumento favorable a la acomodación de los inmigrantes a los hábitos expresivos de los países de acogida en los periodos más recientes de la historia del español, pero no a la pretendida incidencia del contacto interdialectal masivo en los primeros momentos de la colonización. De hecho, en el español clásico, las diferencias entre un grupo y otro figuran entre las más reducidas de todas.

En resumen, el estudio emprendido en estas páginas parece demostrar que, pese a las diferentes restricciones estructurales que han condicionado la variación en las distintas construcciones relacionadas con el queísmo, el perfil extralingüístico de este ha sido-y sigue siendo-mucho más uniforme. De este 
modo, hemos comprobado que, junto a una extensión dialectal relativamente coincidente, el fenómeno presenta un nítido perfil sociolectal y estilístico, que, además, se ha ido consolidando con el paso del tiempo y con independencia de las categorías sintácticas afectadas. Según este, tanto los representantes de los estratos sociales bajos como los textos más cercanos al polo de la inmediatez comunicativa han sido los principales aliados del queísmo en la historia, como corresponde a un fenómeno vernáculo al que, en sentido contrario, se han resistido en mayor medida las élites sociales, sobre todo, en los textos menos íntimos y personales.

\subsection{Variación idiolectal: el comportamiento de los individuos ante el queísmo}

Junto a la actuación de los grupos sociales considerados en el apartado anterior, en la presente investigación hemos querido también comprobar el comportamiento de algunos individuos ante el queísmo. Para ello, en el corpus histórico hemos puesto el foco en aquellos escritores que aparecen mejor representados, con muestras suficientes de la variable lingüística como para poder valorar sus preferencias. El objetivo era doble: por un lado, evaluar, en la medida de lo posible, las preferencias de estos individuos hacia cada una de las variantes implicadas; $y$, por otro, examinar el grado de congruencia de tales preferencias con las tendencias mayoritarias observadas en la época en que escribieron sus textos.

Del resultado de ese estudio, se derivan algunas conclusiones cuando menos sugerentes. La primera de ellas es que, en todas las épocas analizadas, los escritores que siguen las tendencias de variación imperantes son mayoría. Ahora bien, hemos visto también cómo algunas de esas tendencias podían diferir significativamente entre unas construcciones sintácticas y otras. Por ejemplo, entre los autores que escriben cartas y diarios durante la mayor parte del siglo XVI, las soluciones queístas son aplastantes en todos los casos. Sin embargo, a partir del final de esa centuria y, sobre todo, durante todo el siglo XVII, se aprecia una clara divergencia entre las cifras correspondientes a los complementos de categorías nominales (mucho más favorables a las inserciones preposicionales) y el resto, donde las variantes cero continúan siendo claramente mayoritarias. Este comportamiento es, exactamente, el que hemos comprobado en el nivel comunitario durante el siglo XVII, por lo que cabe concluir que, efectivamente, muchos escritores de ese periodo pueden caracterizarse como contemporizadores, ya que respaldan las tendencias centrales de variación imperantes en cada caso.

Ahora bien, junto a estos resultados, hemos observado también la presencia de algunos individuos cuyos usos van más allá de las tendencias distribucionales 
dominantes en su época. Estos pueden ser de dos tipos. Por un lado, se encuentran aquellos escritores que podríamos calificar como conservadores, por su particular fidelidad a las variantes tradicionales queístas, especialmente en periodos en los que la pugna entre estas y las variantes preposicionales estaba ya mucho más equilibrada o era claramente favorable a estas últimas. Un ejemplo de este perfil conservador lo encontramos, por ejemplo, en los miembros de una pequeña red social integrada por el matrimonio Sorolla: Joaquín, el célebre pintor valenciano, su mujer, Clotilde, y el amigo de ambos, el también valenciano Pedro Gil. Entre finales del siglo XIX y las primeras décadas del XX, estos individuos intercambiaron centenares de cartas en las que se aprecia una decidida preferencia por las expresiones queístas, que, en algunos casos (sobre todo, en la correspondencia escrita por Pedro Gil) adquieren magnitudes prácticamente categóricas. Asimismo, este perfil conservador se vislumbra también por las mismas fechas en algunos representantes de las clases subalternas, que llevan las elisiones hasta extremos ya superados (colectivamente) en la época.

Como contrapartida, en los textos encontramos también algunos ejemplos más aislados de escritores que lideran las innovaciones, y se colocan muy por encima del resto en el empleo de las variantes alternativas. Como cabía esperar, las muestras de este trazo idiolectal son especialmente visibles en la etapa contemporánea. Así, algunas figuras destacadas del primer tercio del siglo XX, como el político Manuel Azaña o el escritor modernista Salvador Rueda, muestran una abrupta preferencia por las formas preposicionales en sus escritos, una tendencia que, además, se observa en todas las categorías sintácticas estudiadas. Con todo, ya en el siglo XVIII contamos con algún ejemplo similar, como demuestran las cartas del político asturiano Pedro Rodríguez Campomanes, cuya inclinación por las formas preposionales, aunque no tan radical como en los casos anteriores, es también manifiesta en toda su correspondencia privada.

No obstante, es difícil encontrar líderes de las innovaciones que afecten a la variable lingüística en todas las construcciones. Por el contrario, es más frecuente tropezar con individuos que se colocan en la avanzadilla de estas novedades en ciertas estructuras sintácticas, al tiempo que contemporizan con las tendencias centrales en las demás. Los ejemplos de este perfil idiolectal híbrido son más numerosos en todo el eje temporal, incluso ya desde el siglo XVII. Así, en esta centuria hemos visto cómo diversos representantes de las élites sociales llevaban ciertos usos preposicionales más allá de lo esperable en la época, mientras que se mantenían dentro de la ortodoxia queísta en otros. Entre estos figuran aristócratas como Diego de Silva, y algunas mujeres influyentes, como las monjas abadesas María Jesús de Ágreda y Sor Ángela de la Concepción, o la también religiosa y poetisa mística extremeña Luisa de Carvajal y Mendoza. Mientras que las formas preposicionales son predominantes en su correspondencia en las com- 
pletivas dependientes de sustantivos, adjetivos y verbos, los usos queístas son muy mayoritarios entre las locuciones y oraciones de relativo, y en algunos casos, incluso, de manera prácticamente categórica.

\subsection{Reflexiones finales}

El estudio pancrónico emprendido en esta investigación asume que, para analizar cabalmente la profundidad histórica de un fenómeno de variación sintáctica como el queísmo, deben concitarse diversos requisitos metodológicos: a) la compilación de corpus suficientemente amplios y representativos de periodos antiguos de la historia; b) la concepción oralizante de sus textos; c) la comparación de diversas magnitudes derivadas de un análisis de regresión logística de efectos mixtos, con los que medir el modo en que la variación y el cambio lingüístico han discurrido en el transcurso del tiempo, y d) la aplicación del principio de responsabilidad ante los datos (accountability), esto es, la atención no solo a la variante sobre la que se fija el interés, sino también de aquella o aquellas que podrían haber figurado en su lugar.

Creemos que la aplicación de estos principios ha contribuido a hacernos una idea más precisa acerca de la evolución del queísmo durante los últimos 500 años de la historia del español. En esta evolución, hemos comprobado cómo las distintas construcciones sintácticas analizadas siguen en muchos casos patrones evolutivos divergentes, y a partir de condicionamientos internos también en buena medida diferentes. Ello no impide, sin embargo, que el fenómeno haya mostrado también ciertas regularidades, especialmente en el plano sociolectal, en el que la variable adquiere tintes cercanos a los de un marcador sociolingüístico, dada su especial sensibilidad a la variación diastrática y diafásica durante buena parte de la historia.

Al fortalecimiento de estas conclusiones ha contribuido también la comparación de los datos del corpus histórico con los obtenidos a partir de materiales del habla oral contemporáneos. Esa comparación no solo ha permitido ver las principales líneas de continuidad y ruptura con el pasado, sino también las posibilidades metodológicas que entraña trabajar con textos que, aun siendo de naturaleza diferente, parten de una similar concepción de la lengua, de naturaleza básicamente oral, y alejada de las restricciones que impone la escritura en otras tradiciones más formales. En este sentido, una línea de investigación sugerente que se abre ante nosotros es la de comprobar, con los mismos mimbres teóricos y metodológicos utilizados en esta investigación, cómo reaccionaron esas tradiciones discursivas ante los patrones de variación y cambio lingüístico que se advertían, coetáneamente, en la lengua vernácula. 
